\title{
ARE THE BULGE C-STARS RELATED TO THE SAGITTARIUS DWARF GALAXY?
}

\author{
YUEN KEONG NG \\ Padova Astronomical Observatory \\ Vicolo dell'Osservatorio 5, I-35122 Padova, ITALY
}

When the Sagittarius dwarf galaxy (SDG) crosses the galactic plane star formation might occur in its tidal tail. Carbon stars, due to their brightness, are ideal tracers for such an event. Ng \& Schultheis (1997) suggested that the Bulge carbon stars (Azzopardi et al. 1991; ALRW) might be at SDG's distance. In the galactic Bulge these stars are completely different from carbon stars found in the LMC, SMC and dwarf spheroidal galaxies. If one keeps these stars in the Bulge and tries to explain their properties through a binary evolution scenario, one has to explain why they are not found up to the red giant branch tip: there is a $1^{m} .5$ discrepancy. $\mathrm{Ng}(1997)$ argues that part of the mystery concerning the luminosity of the ALRW stars would be solved if they are related to the SDG. It is demonstrated that the carbon star sequence is in that case similar to the observed SMC sequence. The carbon stars are in that case not metal-rich as previously thought, but they have a metallicity comparable to the LMC, with an age between 0.1-1 Gyr. A significant fraction of the carbon stars still have luminosities fainter than the lower LMC limit of $M_{\mathrm{bol}} \simeq-3^{m} \cdot 5$. A similar trend is present among carbon stars found in other dwarf spheroidals. A scenario through binary evolution is assumed for these stars, because TPAGB models cannot explain their origin through a single-star evolution scenario, even if they form immediately after entering the TP-AGB phase. Acknowledgements for financial support received from the ASI.

\section{References}

Azzopardi M., Lequeux J., Robeirot E., \& Westerlund B.E. 1991, $A \& A S$, 88, 265 (ALRW) $\mathrm{Ng}$ Y.K. 1997, $A \mathscr{E} A$, in press

Ng Y.K., \& Schultheis M. 1997, $A \& A S, \mathbf{1 2 3}, 115$ 